Original

\title{
Visfatin levels in gingival crevicular fluid and serum before and after non-surgical treatment for periodontal diseases
}

\author{
Çiğdem Coşkun Türer1), Umut Balli1), Berrak Güven²), \\ Burcu Özkan Çetinkaya³), and Gonca Çayır Keleş3)

\begin{abstract}
1)Department of Periodontology, Faculty of Dentistry, Bülent Ecevit University, Zonguldak, Turkey
2)Department of Biochemistry, Faculty of Medicine, Bülent Ecevit University, Zonguldak, Turkey

3)Department of Periodontology, Faculty of Dentistry, Ondokuz Mayis University, Samsun, Turkey
\end{abstract}

(Received February 9, 2016; Accepted May 11, 2016)

\begin{abstract}
The purpose of this study was to evaluate visfatin levels at different stages of periodontal disease and in healthy tissues. In addition, the effect of non-surgical periodontal therapy on visfatin levels in gingival crevicular fluid and serum was investigated. Forty-five patients were divided into three groups based on clinical and radiographical findings. Group 1 comprised periodontally healthy individuals $(n=15)$; group 2 comprised patients with gingivitis $(n=15)$; and group 3 was composed of patients with generalized chronic periodontitis $(n=15)$. Gingival crevicular fluid and serum samples were collected before treatment and at 1, 3, and 6 months after treatment. Visfatin levels were measured by enzymelinked immunosorbent assays. Gingival crevicular fluid and serum visfatin levels were higher in patients with chronic periodontitis than those with gingivitis or healthy controls $(P<0.016)$. In addition, visfatin levels were higher in the gingivitis group than in healthy controls $(P<0.016)$. Non-surgical periodontal treatment resulted in a significant reduction in gingival crevicular fluid and serum visfatin levels. Furthermore, visfatin levels increased with inflammation and decreased following periodontal treatment. Our findings suggest that visfatin is an inflammatory
\end{abstract}

Correspondence to Dr. Çiğdem Coşkun Türer, Department of Periodontology, Faculty of Dentistry, Bülent Ecevit University, 67600-Kozlu, Zonguldak, Turkey

Fax:+090-3722613603 E-mail: cigdemturer@gmail.com

Color figures can be viewed in the online issue at J-STAGE. doi.org/10.2334/josnusd.16-0116

DN/JST.JSTAGE/josnusd/16-0116 biomarker of periodontal disease. (J Oral Sci 58, 491-499, 2016)

Keywords: visfatin; periodontal disease; gingival crevicular fluid; serum; post-treatment.

\section{Introduction}

Periodontal disease is a complex biological process involving interactions between microorganisms and the immune/inflammatory response of the host (1). Periodontal destruction is mediated by locally produced proinflammatory cytokines in response to bacterial infection (2). Inappropriate inflammatory responses to pathogenic infection result in loss of connective tissue attachment and alveolar bone breakdown (3). Gingivitis is a gingival inflammation without the loss of supporting periodontal tissues, while periodontitis is characterized by impaired attachment and loss of bone (4). Inflammatory biomarkers can provide additional information to disease progression beyond standard clinical and radiographic examinations (5).

The genetic, microbiological, environmental, and immunological factors involved in the transition from gingivitis to periodontitis have not been defined. Several cytokines are released in response to periodontal infection (5), and cytokine release measurements are used to determine the local responses to bacterial infection. Many cytokines in the gingival crevicular fluid (GCF) have been suggested as potential markers of periodontal destruction (6). Proinflammatory cytokines, such as IL- 6 and TNF- $\alpha$, are considered to be associated with periodontal inflammation (7). 
Adipose tissue produces a variety of cytokines and inflammatory molecules, commonly referred to as adipo(cyto)kines, which regulate different inflammatory processes and are involved in the pathophysiology of periodontitis. Proinflammatory cytokines, such as TNF- $\alpha$ or IL-6, are overexpressed in the adipose tissue of obese patients and contribute to insulin resistance $(8,9)$. Moreover, adipocyte-derived cytokines interfere with immune processes (10). Levels of these cytokines decrease after weight loss (11).

Visfatin is a $52-\mathrm{kDa}$ molecule secreted primarily by visceral white adipose tissue. It was previously described as pre-B-cell colony-enhancing factor (PBEF) and upregulated the production of the pro- and anti-inflammatory cytokines IL-1 $\beta$, IL-1Ra, IL-6, IL-10, and TNF- $\alpha$ in human monocytes (10). Visfatin was identified in human bone marrow as a cytokine-like molecule that is secreted by activated lymphocytes and stimulates the early stages of B cell formation (12). It is also secreted by neutrophils in response to pathogens and stimulates monocytes to produce inflammatory mediators (10). Visfatin has been associated with several chronic inflammatory diseases, including diabetes mellitus (13), inflammatory bowel disease (10), rheumatoid arthritis (14), acute lung disease (15), and sepsis (16).

Periodontal inflammation increases proinflammatory cytokine production, which may influence visfatin expression in periodontal tissues. This led us to hypothesize that visfatin is a potential biomarker for periodontal diseases.

Non-surgical periodontal therapy is an anti-infective therapy with mechanical and chemotherapeutic approaches to minimize or eliminate the microbial biofilm, which is the primary etiology of gingivitis and periodontitis. Mechanical therapies, such as supragingival and subgingival scaling and root planing, use hand or power-driven scalers and curets (17).

Proinflammatory cytokines associated with periodontal inflammation such as IL- 6 and IL-1 $\beta$ may be related to visfatin expression in periodontal tissues (18). Visfatin has been associated with several chronic inflammatory diseases; one such example is rheumatoid arthritis. Periodontitis and rheumatoid arthritis are chronic inflammatory conditions with similar pathological features (19). We evaluated visfatin levels in GCF and serum in periodontally healthy tissue and at different stages of periodontal disease to test the hypothesis that visfatin is as a diagnostic biomarker and increases as periodontal disease progresses. In addition, we quantified the effect of non-surgical periodontal therapy on visfatin levels in GCF and serum 1, 3, and 6 months after treatment.
Furthermore, we tested the correlations between visfatin levels and clinical parameters.

\section{Materials and Methods}

\section{Study population}

This study investigated a population of individuals who came to the Department of Periodontology at Bulent Ecevit University. Forty-five participants were recruited and included 22 women and 23 men aged 25-45. The study was designed in accordance with the rulings of the declaration of Helsinki and the Ethics Committee of Ondokuz Mayis University (2012/568). Informed written consent was provided by every participant.

\section{Inclusion criteria}

The participants were separated into three groups depending on the periodontal parameters as follows: Group 1 contained individuals with a clinically healthy periodontium $(n=15)$, group 2 was composed of patients with gingivitis $(n=15)$, and group 3 contained patients with generalized chronic periodontitis $(n=15)$. A classification system published in 1999 was utilized for disease characterization (4).

A clinical full-mouth examination and a radiographic periodontal examination were performed before participation in the study. The plaque index (PI) (20), bleeding on probing (BOP) (21), gingival index (GI) (22), and probing pocket depth (PPD) were measured during this examination. All participants had at least 20 teeth, not including their third molars. According to the examination, group 1 had no indications of inflammation, no loss of attachment, or loss of bone (GI $=0, \mathrm{PPD} \leq 3 \mathrm{~mm}$, $\mathrm{CAL} \leq 3 \mathrm{~mm}$ ). Individuals in group 2 showed signs of inflammation, such as redness, higher BOP levels, and edema. However, no attachment loss or alveolar bone loss were observed (GI $\geq 1$, PPD and $\mathrm{CAL} \leq 3 \mathrm{~mm}$ ). Individuals in group 3 showed clinical signs of inflammation, GI $>1, \mathrm{PPD} \geq 5 \mathrm{~mm}, \mathrm{CAL} \geq 5 \mathrm{~mm}$, and alveolar bone loss affecting $>30 \%$ of the existing teeth. A Williams periodontal probe was used to take all the measurements (Hu-Friedy, Chicago, IL, USA) and all measurements were made by one person. Ten people were chosen at random to calibrate the measurements beforehand. These people were assessed on two separate occasions, with two days separating each of the measurements. These findings were satisfactorily reproducible, the baseline measurements and measurements taken after $48 \mathrm{~h}$ were within $10 \%$ of each other on the mm scale (23).

\section{Exclusion criteria}

Individuals with diseases that could influence visfatin 
expression such as rheumatoid arthritis, diabetes mellitus, cancer, or obesity, were excluded. Body mass index (BMI) was used to determine obesity and was calculated in kilograms divided by height in meters squared. Participants with a BMI greater than 24.9 were excluded. Further exclusion criteria were pregnancy, lactation, history of medications that could impact periodontal status, aggressive periodontitis, smoking, tobacco use, and periodontal care within the last half year. Each participant was questioned directly about tobacco use.

\section{Collecting GCF and site selection}

To prevent contamination of samples with blood, the samples were collected by a single researcher (CCT) a day after the measurements were taken. A single sampling site was set for each individual, and GCF samples were collected from sites with no clinical inflammation in group 1. In group 2, GCF samples were collected from the main site of inflammation displaying redness, edema and BOP without CAL. GCF samples were taken from sites with the most significant inflammation and CAL in group 3.

Supragingival plaque was managed at baseline, 1 month, 3 months, and 6 months after periodontal therapy if needed. To prevent contamination from saliva, samples were collected with the use of cotton rolls, and GCF samples were taken after the site had been carefully dried using the intracrevicular method with filter paper (Periopaper, ProFlow, Inc., Amityville, NY, USA) (24). The strips were inserted into the crevice until resistance was felt and put to one side in stasis for $30 \mathrm{~s}$. Electronic impedance was used to determine the GCF volume of every strip (Periotron 8000, ProFlow Inc., Hewlett, NY, USA). Strips with traces of blood or saliva were discarded. All strips were placed into an Eppendorf tube and stored at $-40^{\circ} \mathrm{C}$ before assaying.

\section{Serum collection}

Peripheral blood samples were collected $(2 \mathrm{~mL})$ from the antecubital fossa via venipuncture using a sterile syringe, and the serum component was separated by centrifugation for $5 \mathrm{~min}$ at $3,000 \mathrm{~g}$. Serum was stored at $-40^{\circ} \mathrm{C}$.

\section{Periodontal treatment}

The same investigator (CCT) conducted all non-surgical periodontal treatments. Patients in group 3 received non-surgical periodontal therapy along with oral hygiene guidelines once the baseline GCF and the serum samples were collected. Non-surgical periodontal therapy included scaling and root planing (SRP) with manual scalers and curets (Hu-Friedy). SRP was applied quad- rant-by-quadrant over 2 months. A local anesthetic was applied where necessary. Patients in group 2 received non-surgical periodontal treatment for 4 weeks involving scaling along with oral hygiene guidelines. Oral hygiene instructions were provided to group 1 with no additional treatment. All oral hygiene guidelines were in accordance with the modified Bass method and included brushing and dental flossing repeated every 2 weeks. After periodontal therapy, patients were reassessed and serum and GCF samples were collected 1 month, 3 months, and 6 months after treatment. All patients were present for the subsequent assessment. A follow-up examination was performed on all participants and no participant reported the use of antibiotics during the study period.

\section{Assaying visfatin levels}

Sample strip tubes were combined with $100 \mu \mathrm{L}$ phosphate-buffered saline $(\mathrm{pH}$ 7.4) and homogenized for 1 min. Samples were vortexed, then centrifuged for $15 \mathrm{~min}$ at $4^{\circ} \mathrm{C}$ at 3,000 g. Total visfatin levels were measured in the supernatants using a sandwich enzyme-linked immunosorbent assay, using commercially available kits (Hangzhou Eastbiopharm Co. Ltd, Hangzhou, China). All samples were assayed in replicate according to the manufacturer's instructions. The amount of concentration GCF was calculated by dividing the total amount (ng) (the visfatin level in GCF analyzed by ELISA using commercially available kits) by the volume of the GCF $(\mu \mathrm{L})$.

\section{Statistical analysis}

We used the primary outcome variable (the difference in the GCF visfatin levels post-treatment) to calculate the sample size. Predictions and estimations were based on the pilot research study, and ten individuals were used per group. We speculated that a sample population of 11 individuals within all groups would allow a type I error level of $\alpha=0.05$ (5\% probability) and a type II error level of $\beta=0.20$ ( $80 \%$ power). To allow for possible dropouts, we included 15 patients in each group.

To determine normal distribution, the ShapiroWest test was used. Once normality was rejected $(P<$ $0.05)$, the inter-group comparisons were analyzed by nonparametric Kruskal-Wallis test, after which the Mann-Whitney $U$ (Bonferroni-adjusted) test was utilized for a post-hoc comparison $(\alpha=0.05 / 3=0.016$ was considered statistically significant). Inter-group differences in clinical and biochemical parameters at 1, 3 and 6 months post-treatment were assessed using the MannWhitney $U$ test. The paired clinical limitations (between 6 months and baseline) and intra-group comparisons 
Table 1 Clinical parameters at baseline and 6 months (periodontal examination of full-mouth and sampled sites) in the different groups

\begin{tabular}{|c|c|c|c|c|c|c|c|c|c|c|}
\hline & \multicolumn{5}{|c|}{ Baseline } & \multicolumn{5}{|c|}{6 months } \\
\hline & PPD (mm) & CAL (mm) & GI & PI & BOP $(\%)$ & PPD (mm) & $\mathrm{CAL}(\mathrm{mm})$ & $\mathrm{GI}^{\mathrm{c}}$ & $\mathrm{PI}^{\mathrm{c}}$ & $\mathrm{BOP}^{\mathrm{c}}(\%)$ \\
\hline $\begin{array}{l}\text { Full-mouth } \\
\text { group } 1\end{array}$ & $\begin{array}{c}2.07 \pm 0.39 \\
(1.96)\end{array}$ & $\begin{array}{c}2.07 \pm 0.39 \\
(1.96)\end{array}$ & $\begin{array}{c}0.00 \pm 0.00 \\
\quad(0.00)\end{array}$ & $\begin{array}{c}0.41 \pm 0.14 \\
\quad(0.43)\end{array}$ & $\begin{array}{c}0.00 \pm 0.00 \\
(0.00)\end{array}$ & & & & & \\
\hline $\begin{array}{l}\text { Sampled sites } \\
\text { group } 1\end{array}$ & $\begin{array}{c}1.80 \pm 0.41 \\
\quad(2.00)\end{array}$ & $\begin{array}{c}1.80 \pm 0.41 \\
(2.00)\end{array}$ & $\begin{array}{c}0.00 \pm 0.00 \\
\quad(0.00)\end{array}$ & $\begin{array}{c}0.00 \pm 0.00 \\
(0.00)\end{array}$ & $\begin{array}{c}0.00 \pm 0.00 \\
\quad(0.00)\end{array}$ & & & & & \\
\hline $\begin{array}{l}\text { Full-mouth } \\
\text { group } 2\end{array}$ & $\begin{array}{c}2.11 \pm 0.25 \\
\quad(2.16)\end{array}$ & $\begin{array}{c}2.11 \pm 0.25 \\
\quad(2.16)\end{array}$ & $\begin{array}{c}1.64 \pm 0.05^{\mathrm{a}} \\
\quad(1.64)\end{array}$ & $\begin{array}{c}1.05 \pm 0.07^{\mathrm{a}} \\
(1.03)\end{array}$ & $\begin{array}{c}77.79 \pm 7.32^{\mathrm{a}} \\
(78.12)\end{array}$ & $\begin{array}{c}2.03 \pm 0.30 \\
\quad(2.03)\end{array}$ & $\begin{array}{c}2.03 \pm 0.30 \\
\quad(2.03)\end{array}$ & $\begin{array}{c}0.23 \pm 0.16 \\
(0.24)\end{array}$ & $\begin{array}{c}0.35 \pm 0.14 \\
\quad(0.35)\end{array}$ & $\begin{array}{c}11.19 \pm 2.05 \\
\quad(10.58)\end{array}$ \\
\hline $\begin{array}{l}\text { Sampled sites } \\
\text { group } 2\end{array}$ & $\begin{array}{c}2.00 \pm 0.76 \\
\quad(2.00)\end{array}$ & $\begin{array}{c}2.00 \pm 0.76 \\
(2.00)\end{array}$ & $\begin{array}{c}2.40 \pm 0.51^{\mathrm{a}} \\
\quad(2.00)\end{array}$ & $\begin{array}{c}2.47 \pm 0.52^{\mathrm{a}} \\
\quad(2.00)\end{array}$ & $\begin{array}{c}100.00 \pm 0.00^{\mathrm{a}} \\
\quad(100.00)\end{array}$ & $\begin{array}{c}1.60 \pm 0.51 \\
(2.00)\end{array}$ & $\begin{array}{c}1.60 \pm 0.51 \\
(2.00)\end{array}$ & $\begin{array}{c}0.33 \pm 0.49 \\
(0.00)\end{array}$ & $\begin{array}{c}0.13 \pm 0.35 \\
\quad(0.00)\end{array}$ & $\begin{array}{c}1.33 \pm 0.49 \\
(1.00)\end{array}$ \\
\hline $\begin{array}{l}\text { Full-mouth } \\
\text { group } 3\end{array}$ & $\begin{array}{c}4.56 \pm 0.43^{\mathrm{ab}} \\
\quad(4.44)\end{array}$ & $\begin{array}{c}5.80 \pm 0.34^{\mathrm{ab}} \\
\quad(5.78)\end{array}$ & $\begin{array}{c}2.53 \pm 0.18^{\mathrm{ab}} \\
(2.63)\end{array}$ & $\begin{array}{c}2.28 \pm 0.20^{\mathrm{ab}} \\
(2.26)\end{array}$ & $\begin{array}{c}88.80 \pm 3.58^{\mathrm{ab}} \\
(88.61)\end{array}$ & $\begin{array}{c}2.50 \pm 0.22^{\mathrm{bc}} \\
(2.51)\end{array}$ & $\begin{array}{c}3.30 \pm 0.52^{\mathrm{bc}} \\
(3.33)\end{array}$ & $\begin{array}{c}0.55 \pm 0.20^{\mathrm{b}} \\
(0.56)\end{array}$ & $\begin{array}{c}0.46 \pm 0.21 \\
\quad(0.47)\end{array}$ & $\begin{array}{c}11.32 \pm 2.65 \\
(11.26)\end{array}$ \\
\hline $\begin{array}{l}\text { Sampled sites } \\
\text { group } 3\end{array}$ & $\begin{array}{c}6.20 \pm 0.77^{\mathrm{ab}} \\
(6.00)\end{array}$ & $\begin{array}{c}7.13 \pm 0.83^{\mathrm{ab}} \\
(7.00)\end{array}$ & $\begin{array}{c}2.53 \pm 0.52^{\mathrm{a}} \\
(3.00)\end{array}$ & $\begin{array}{c}2.13 \pm 0.35^{\mathrm{a}} \\
(2.00)\end{array}$ & $\begin{array}{c}100.00 \pm 0.00^{\mathrm{a}} \\
\quad(100.00)\end{array}$ & $\begin{array}{c}2.67 \pm 0.49^{\mathrm{bc}} \\
(3.00)\end{array}$ & $\begin{array}{c}3.60 \pm 0.51^{\mathrm{bc}} \\
(4.00)\end{array}$ & $\begin{array}{c}0.40 \pm 0.51 \\
(0.00)\end{array}$ & $\begin{array}{c}0.33 \pm 0.49 \\
(0.00)\end{array}$ & $\begin{array}{c}1.13 \pm 0.35 \\
(1.00)\end{array}$ \\
\hline
\end{tabular}

were analyzed using the Wilcoxon signed-rank test for the periodontitis and gingivitis groups. The Freidman test was used to determine significant differences in biochemical parameters in group 2 and group 3 at baseline, 1, 3, and 6 months. To assess the significance of pairwise differentiation, the Wilcoxon signed-rank test was used with Bonferroni correction to account for multiple comparisons. For comparison of paired periodontal parameters (baseline and 6 month), $\alpha=0.05$ was applied to determine statistical significance, and for comparison of paired biochemical parameters (at baseline and 1,3 , and 6 months) $P=0.05 / 6=0.008$ was applied to determine statistical significance. $\chi^{2}$ analysis determined the percentage of genders among the groups and the BOP percentage. Spearman's rank correlation test was used to test that the relationship between the total volume of visfatin and the visfatin serum levels were within clinical periodontal limits. All tests were performed using SPSS, and $P<0.05$ indicated statistical significance (SPSS Inc., version 19.0, Chicago, IL, USA).

\section{Clinical results}

\section{Results}

Table 1 summarizes the clinical findings. PPD, BOP, GI, PI, and CAL are provided. All clinical parameters were significantly greater in group 2 and group 3 compared with group 1 (Kruskal-Wallis/Bonferroni-adjusted test, Mann-Whitney $U, P<0.016)$. There were no significant differences in sex, age (Kruskal-Wallis, Chi-square test, $P>0.05$ ), or BMI (Kruskal-Wallis, $P>0.05$ ) of participants between groups (Table 2). Six months after SRP treatment finished, the clinical parameters were significantly reduced with regard to full-mouth and sample site examinations (Mann-Whitney $U, P<0.05$ ).
Table 2 Age, gender distribution and BMI scores in the different groups

\begin{tabular}{lccc}
\hline Parameter & Group 1 & Group 2 & Group 3 \\
\hline Age (years) & & & \\
Mean \pm SD & $34.80 \pm 4.5$ & $35.20 \pm 4.4$ & $38.73 \pm 4.3$ \\
Median & 36 & 36 & 38 \\
Gender & & & \\
Males & 7 & 8 & 8 \\
Females & 8 & 7 & 7 \\
BMI $^{\mathrm{b}}$ & & & \\
Mean \pm SD & $22.43 \pm 1.5$ & $23.11 \pm 1.1$ & $22.39 \pm 1.5$ \\
Median & 22.65 & 23.06 & 22.48 \\
\hline
\end{tabular}

Data are expressed as the mean \pm standard deviation. ${ }^{\text {aKuskal-Wallis, }}$ $P>0.05$; ${ }^{\mathrm{b}}$ Chi-square tests, $P>0.05$.

\section{Biochemical results}

Visfatin was detected in all GCF and serum samples. Figures 1 and 2 depict the total amounts and concentration amounts of visfatin in GCF, respectively. In group 3, GCF visfatin levels were statistically higher compared with group 1 and group 2 (Bonferroni-adjusted Mann-Whitney $U$ test, $P<0.016)$. Furthermore, GCF visfatin levels were reduced in the healthy participants compared with gingivitis patients. Serum visfatin levels were also significantly reduced in group 1 and group 2 compared with group 3 (Fig. 3). (Bonferroni-adjusted Mann-Whitney $U$ test, $P<0.016)$. Serum visfatin levels were significantly higher in group 2 compared group 1 (Bonferroni-adjusted Mann-Whitney $U$ test, $P<0.016$ ). Serum levels are consistent with visfatin levels detected in GCF. Figure 4 depicts the relationship between visfatin levels and periodontal disease.

\section{Impact of periodontal therapy on visfatin levels} Periodontal treatment with SRP significantly reduced 


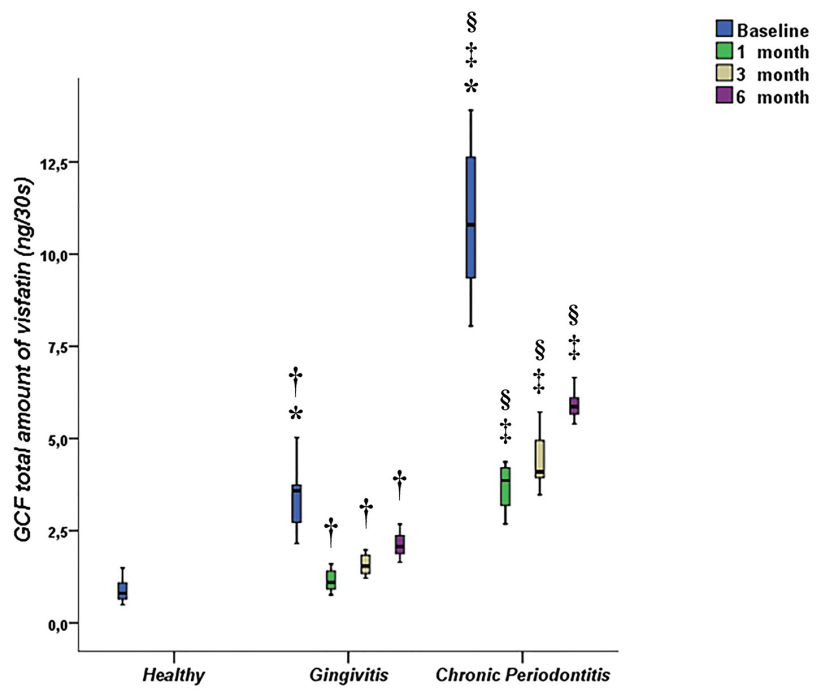

Fig. 1 Total amount of visfatin in GCF in different groups. *Statistically significant difference from healthy (Bonferroni-adjusted Mann-Whitney $U$ test); †Statistically significant difference among time frame in gingivitis group (Bonferroni-adjusted Wilcoxon signed-rank test); $\$$ Statistically significant difference among time frame in chronic periodontitis group (Bonferroni-adjusted Wilcoxon signed-rank test); §Statistically significant difference from gingivitis group (Bonferroni-adjusted Mann-Whitney $U$ test). Data are presented as box and whisker plots. The median value is indicated by the line within the box plot. The box extends from the 25 th to the 75th percentiles. Whiskers extend to show the highest and lowest values.

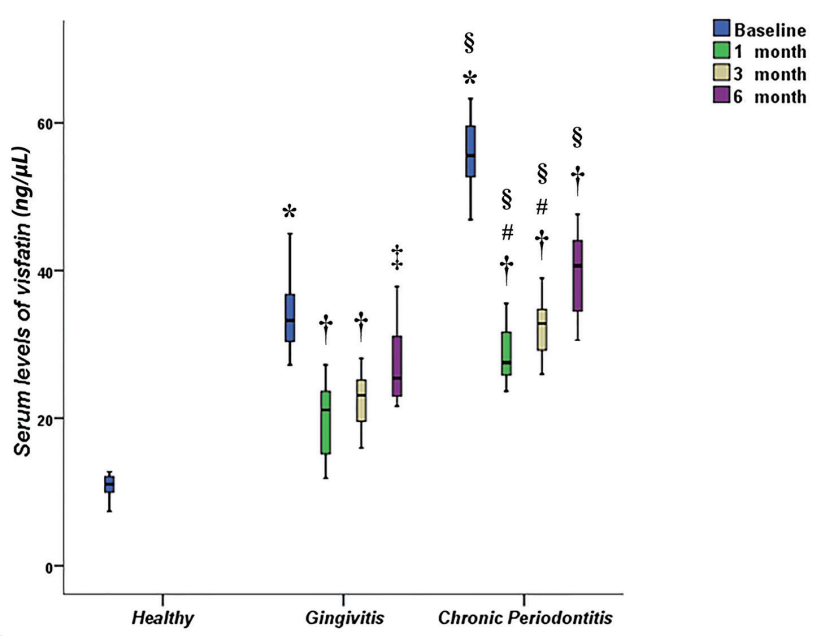

Fig. 3 Serum levels of visfatin in different groups. *Statistically significant difference from healthy (Bonferroni-adjusted MannWhitney $U$ test); $\uparrow$ Statistically significant difference from baseline (Bonferroni-adjusted Wilcoxon signed-rank test); †Statistically significant difference from 1 month examination (Bonferroni-adjusted Wilcoxon signed-rank test); \#Statistically significant difference from 6 Month examination (Bonferroni-adjusted Wilcoxon signed-rank test); §Statistically significant difference from gingivitis group (Bonferroni-adjusted Mann-Whitney $U$ test). Data are presented as box and whisker plots. The median value is indicated by the line within the box plot. The box extends from the 25th to the 75th percentiles. Whiskers extend to show the highest and lowest values.

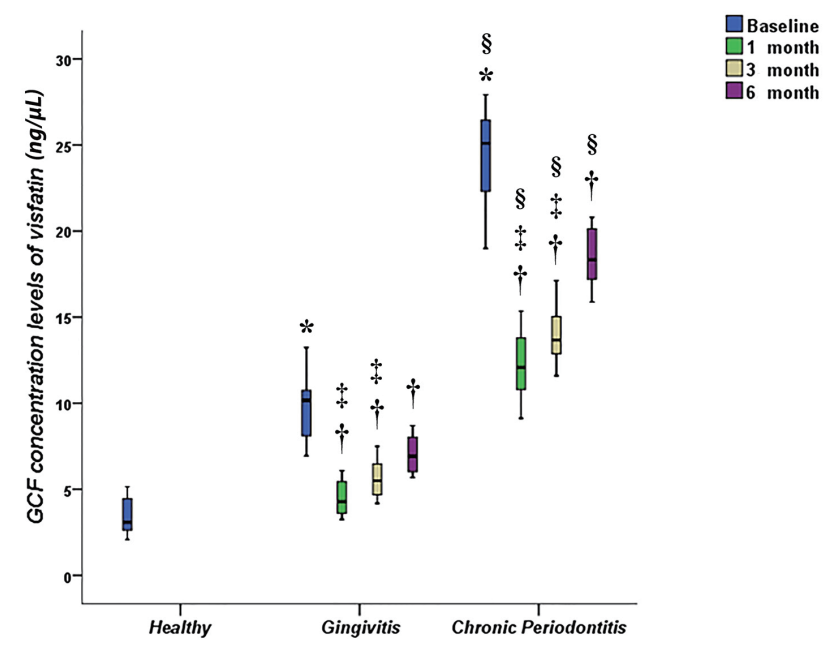

Fig. 2 Concentration levels of visfatin in GCF in different groups. *Statistically significant difference from healthy (Bonferroniadjusted Mann-Whitney $U$ test); † Statistically significant difference from baseline (Bonferroni-adjusted Wilcoxon signed-rank test); tStatistically significant difference from 6 month examination (Bonferroni-adjusted Wilcoxon signed-rank test); §Statistically significant difference from gingivitis group (Bonferroni-adjusted Mann-Whitney $U$ test). Data are presented as box and whisker plots. The median value is indicated by the line within the box plot. The box extends from the 25 th to the 75 th percentiles. Whiskers extend to show the highest and lowest values.

visfatin levels in GCF and serum (Bonferroni-adjusted Wilcoxon signed-rank test, $P<0.008)$. GCF visfatin levels were statistically significant between group 2 and the group 3 (Bonferroni-adjusted Wilcoxon signed-rank test, $P<0.008$ ) (Fig. 1). Following SRP treatment, visfatin levels were significantly different between group 2 and group 3 (Bonferroni-adjusted Wilcoxon signed-rank test, $P<0.008$ ) at baseline, 1 and 3 months. A significant reduction in serum visfatin levels were observed 6 months after SRP in group 3. No significant difference was observed in group 2 (Bonferroni-adjusted Wilcoxon signed-rank test, $P>0.008)$ (Fig. 3).

\section{Correlations}

Correlation coefficients are presented in Table 3. A significant positive correlation was discovered between the total amount of visfatin and the serum visfatin in group 3 (Spearman's rank correlation, $P<0.05$ ). CAL and GI correlated positively with the total amounts of visfatin and serum visfatin levels in group 3 (Spearman's rank correlation, $P<0.05$ ). Total visfatin levels and GI correlated positively in group 2 (Spearman's rank correlation, $P<0.05)$. No correlation was observed between serum visfatin levels and CAL or GI in group 1 and group 

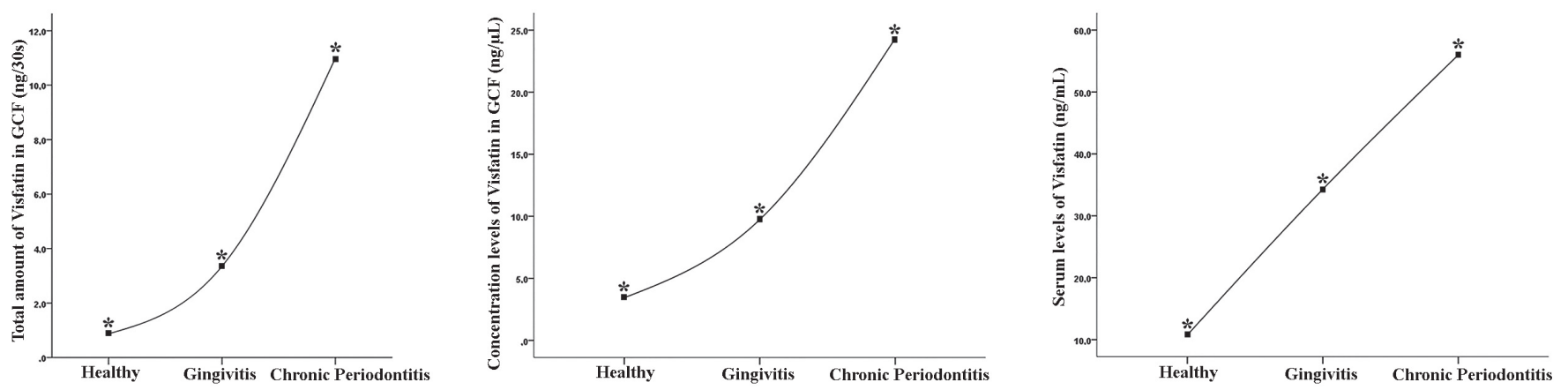

Fig. 4 Visfatin levels in GCF and serum. *Statistically significant difference among all groups (Kruskal-Wallis/Bonferroni-adjusted MannWhitney $U$ ).

Table 3 Spearman's rank correlation (r) among groups with respect to TA-visfatin, S-visfatin, number of deep sites, CAL, and GI

\begin{tabular}{|c|c|c|c|c|c|c|c|c|c|c|}
\hline & & $\begin{array}{l}\text { TA-Visfatin } \\
\text { to S-Visfatin }\end{array}$ & $\begin{array}{c}\text { TA-Visfatin to } \\
4 \leq \mathrm{PPD} \leq 5\end{array}$ & $\begin{array}{l}\text { TA-Visfatin } \\
\text { to } P P D \geq 6\end{array}$ & $\begin{array}{l}\text { S-Visfatin to } \\
4 \leq \mathrm{PPD} \leq 5\end{array}$ & $\begin{array}{l}\text { S-Visfatin to } \\
\mathrm{PPD} \geq 6\end{array}$ & $\begin{array}{c}\text { TA-Visfatin } \\
\text { to CAL }\end{array}$ & $\begin{array}{l}\text { S-Visfatin to } \\
\text { CAL }\end{array}$ & $\begin{array}{c}\text { TA-Visfatin } \\
\text { to GI }\end{array}$ & $\begin{array}{l}\text { S-Visfatin } \\
\text { to GI }\end{array}$ \\
\hline \multirow{2}{*}{ Group 1} & $\mathrm{r}$ & 0.445 & NA & NA & NA & NA & 0.424 & 0.347 & NA & NA \\
\hline & $P$ & 0.096 & NA & NA & NA & NA & 0.115 & 0.204 & NA & NA \\
\hline \multirow{2}{*}{ Group 2} & $\mathrm{r}$ & 0.493 & NA & NA & NA & NA & 0.465 & 0.444 & $0.535^{*}$ & 0.472 \\
\hline & $P$ & 0.062 & NA & NA & NA & NA & 0.081 & 0.098 & $0.040^{*}$ & 0.075 \\
\hline \multirow{2}{*}{ Group 3} & $\mathrm{r}$ & $0.561^{*}$ & $0.549^{*}$ & $0.577 *$ & 0.493 & $0.520^{*}$ & $0.627 *$ & $0.522 *$ & $0.650^{*}$ & $0.526^{*}$ \\
\hline & $P$ & $0.030^{*}$ & $0.034 *$ & $0.024 *$ & 0.062 & $0.047 *$ & $0.012 *$ & $0.046^{*}$ & $0.034 *$ & $0.044 *$ \\
\hline \multirow{2}{*}{ All groups } & $\mathrm{r}$ & $0.945^{*}$ & NA & NA & NA & NA & $0.811^{*}$ & $0.802 *$ & NA & NA \\
\hline & $P$ & $0.000^{*}$ & NA & NA & NA & NA & $0.000^{*}$ & $0.000 *$ & NA & NA \\
\hline
\end{tabular}

TA-Visfatin: Total amount of visfatin; S-Visfatin: Serum levels of visfatin; *Statistically significant $(P<0.05)$; NA: Not applicable.

2 (Spearman's rank correlation, $P>0.05$ ). No significant correlation was observed between total visfatin levels and CAL or serum visfatin levels (Spearman's rank correlation, $P>0.05$ ).

Significant positive correlations were found between total visfatin levels and the number of areas with $4 \leq$ $\mathrm{PPD} \leq 5 \mathrm{~mm}$, the serum visfatin levels and the number of areas with PPD $\geq 6 \mathrm{~mm}$, as well as the total amounts of visfatin and the number of areas with PPD $\geq 6 \mathrm{~mm}$ (the Spearman's rank correlation, $P<0.05$ ). No correlation was seen between the number of sites with $4 \leq \mathrm{PPD} \leq$ $5 \mathrm{~mm}$ and serum visfatin levels (the Spearman's rank correlation, $P>0.05$ ).

\section{Discussion}

We assessed the GCF and serum visfatin levels in periodontal disease and following periodontal treatment. Visfatin levels were lower in healthy fluid compared with periodontally diseased fluid. Furthermore, elevated visfatin levels in the gingivitis group decreased after periodontal treatment.

Increasing evidence suggests that inflammation increases visfatin levels in a number of diseases, including diabetes mellitus (13), inflammatory bowel disease (10), rheumatoid arthritis (14), acute lung disease (15), and sepsis (16). Innate immunity and inflammation is dependent on visfatin (25). Periodontal diseases characterized by chronic inflammation are among the most common conditions in humans worldwide (26).

Periodontal disease has many causes and has been associated with variable systemic diseases and obesity $(10,14,15)$. In this study, participants were selected from a population of healthy, non-obese, non-smoking individuals. Subjects were chosen from a particular age range due to the chronic nature of the disease. To avoid an effect of gender on visfatin levels, an equal number of males and females were tested per group.

GCF may contain potential biomarkers for periodontal and systematic diseases $(27,28)$. PD activity can be determined by inflammatory molecules within GCF (29). We used paper strips to collect GCF, which is inexpensive and can be used for specific locations. Furthermore, this approach produced minimal discomfort for the participant (24). During the collection of GCF, particular care was taken to safeguard the sulcular epithelium.

The amount of concentration GCF was calculated by dividing the total amount (ng) (the visfatin level in GCF analyzed by ELISA using commercially available kits) by the volume of the GCF $(\mu \mathrm{L})$. Calculating the total amount in GCF rather than a concentration is 
more relevant to the connection between periodontal disease and GCF components (30,31). Additionally, total cytokines better indicate the constituent activity of GCF than the concentration because the sample volume directly impacts the concentration (32). Herein, data are considered on the basis of total amounts.

Serum components indicate the level of inflammatory response to periodontal pathogens (33). We investigated serum visfatin levels in the present study before and after periodontal treatment.

Pradeep et al. reported that GCF and serum visfatin concentrations increase with severity of periodontal disease and visfatin was described as an inflammatory marker in periodontal disease (18). Mohamed et al. reported a positive correlation between PD and GCF visfatin levels (34). Visfatin levels were also increased in the saliva following periodontal inflammation $(35,36)$. These findings support our results that visfatin levels correlated positively with the severity of periodontitis. In addition, we evaluated visfatin levels after non-surgical periodontal treatment. A previous study showed that visfatin levels decreased 8 weeks after periodontal treatment (37). Wu et al. (38) evaluated the role of visfatin in patients with T2DM and CP before and 3 and 6 months after non-surgical periodontal treatment. They reported that non-surgical periodontal treatment reduced visfatin and $\mathrm{HbAlc}$ levels in periodontitis patients with T2DM. The serum and salivary levels of visfatin were also shown to decrease significantly after non-surgical periodontal treatment (39). In the present study, a significant decrease in visfatin levels was observed 1 month after non-surgical periodontal treatment in gingivitis and $\mathrm{CP}$ individuals. This reduction was smaller after 3 and 6 months. Özcan et al. (40) showed that $P$. gingivalis infection in the periodontal pockets and EBV in plaque increase visfatin secretion. We evaluated the correlation between visfatin levels and periodontal pocket depth and found that these were positively correlated. Similarly, Mohamed et al. reported that visfatin increased significantly as the number of diseased sites increased.

This study has demonstrated that visfatin levels were greater in $\mathrm{CP}$ and gingivitis patients compared with controls and were higher in $\mathrm{CP}$ patients than gingivitis patients. This indicates that visfatin may be secreted in response to inflammation. One month after treatment with SRP, a significant reduction in visfatin levels was seen in group 2 and group 3 . This study demonstrated that visfatin levels are reduced by periodontal treatment and that this reduced inflammation. Reduced inflammation may be associated with altered visfatin levels. A small increase in visfatin was found 3 and 6 months after SRP in gingivitis and CP patients. This may be explained by insufficient oral hygiene to control periodontal inflammation. These findings suggested that visfatin levels can increase in response to uncontrolled periodontal inflammation, even after successful non-surgical periodontal treatment.

We also examined the correlation between visfatin levels and two different periodontal pocket depth ranges. We found a positive and significant correlation between visfatin levels in GCF with the number of sites $4 \leq$ PPD $\leq 5 \mathrm{~mm}$ and PPD $\geq 6 \mathrm{~mm}$. No correlations were found between serum visfatin levels with the number of sites 4 $\leq \mathrm{PPD} \leq 5 \mathrm{~mm}$. Thus, may be due to the localized secretion from the cells response to periodontal inflammation.

In conclusion, visfatin levels significantly increased with the severity of periodontal disease. This suggests that visfatin is a potential inflammatory biomarker of periodontal disease in GCF and serum. Furthermore, visfatin levels decreased after periodontal treatment. This supports the hypothesis that visfatin plays a role in periodontal inflammation. Taken together, our findings show that visfatin may represent a biomarker for periodontal disease susceptibility. Multicenter studies with larger populations are needed to validate these findings.

\section{Acknowledgments}

This work was supported by Research Fund of Ondokuz Mayis University Project (Number: 2013-62550515-04).

\section{Conflict of interest}

No potential conflict of interest relevant to this article was reported.

\section{References}

1. Kornman KS (2008) Mapping the pathogenesis of periodontitis: a new look. J Periodontol 79, Suppl 8, 1560-1568.

2. Van Dyke TE, Serhan CN (2003) Resolution of inflammation: a new paradigm for the pathogenesis of periodontal diseases. J Dent Res 82, 82-90.

3. Kirkwood KL, Cirelli JA, Rogers JE, Giannobile WV (2007) Novel host response therapeutic approaches to treat periodontal diseases. Periodontol 2000 43, 294-315.

4. Armitage GC (1999) Development of a classification system for periodontal diseases and conditions. Ann Periodontol 4, 1-6.

5. Kinane DF, Preshaw PM, Loos BG (2011) Host-response: understanding the cellular and molecular mechanisms of host-microbial interactions--consensus of the seventh European workshop on periodontology. J Clin Periodontol 38, Suppl 11, 44-48.

6. Barros SP, Williams R, Offenbacher S, Morelli T (2016) Gingival crevicular fluid as a source of biomarkers for peri- 
odontitis. Periodontol 2000 70, 53-64.

7. Preshaw PM, Taylor JJ (2011) How has research into cytokine interactions and their role in driving immune responses impacted our understanding of periodontitis? J Clin Periodontol 38, 60-84.

8. Hotamisligil GS, Arner P, Caro JF, Atkinson RL, Spiegelman BM (1995) Increased adipose tissue expression of tumor necrosis factor- $\alpha$ in human obesity and insulin resistance. $\mathrm{J}$ Clin Invest 95, 2409-2415.

9. Fried SK, Bunkin DA, Greenberg AS (1998) Omental and subcutaneous adipose tissues of obese subjects release interleukin-6: depot difference and regulation by glucocorticoid. J Clin Endocrinol Metab 83, 847-850.

10. Moschen AR, Kaser A, Enrich B, Mosheimer B, Theurl M, Niederegger $\mathrm{H}$ et al. (2007) Visfatin, an adipocytokine with proinflammatory and immunomodulating properties. $\mathrm{J}$ Immunol 178, 1748-1758.

11. Ziccardi P, Nappo F, Giugliano G, Esposito K, Marfella R, Cioffi $\mathrm{M}$ et al. (2002) Reduction of inflammatory cytokine concentrations and improvement of endothelial functions in obese women after weight loss over one year. Circulation 105, 804-809.

12. Samal B, Sun Y, Stearns G, Xie C, Suggs S, McNiece I (1994) Cloning and characterization of the cDNA encoding a novel human pre-B-cell colony-enhancing factor. Mol Cell Biol 14, 1431-1437.

13. Dahl TB, Yndestad A, Skjelland M, Øie E, Dahl A, Michelsen A et al. (2007) Increased expression of visfatin in macrophages of human unstable carotid and coronary atherosclerosis: possible role in inflammation and plaque destabilization. Circulation 115, 972-980.

14. Nowell MA, Richards PJ, Fielding CA, Ognjanovic S, Topley N, Williams AS et al. (2006) Regulation of pre-B cell colony-enhancing factor by STAT-3-dependent interleukin-6 trans-signaling: implications in the pathogenesis of rheumatoid arthritis. Arthritis Rheum 54, 2084-2095.

15. Ye SQ, Simon BA, Maloney JP, Zambelli-Weiner A, Gao L, Grant A et al. (2005) Pre-B-cell colony-enhancing factor as a potential novel biomarker in acute lung injury. Am J Respir Crit Care Med 171, 361-370.

16. Jia SH, Li Y, Parodo J, Kapus A, Fan L, Rotstein OD et al. (2004) Pre-B cell colony-enhancing factor inhibits neutrophil apoptosis in experimental inflammation and clinical sepsis. J Clin Invest 113, 1318-1327.

17. Drisko CH (2001) Nonsurgical periodontal therapy. Periodontol 2000 25, 77-88.

18. Pradeep AR, Raghavendra NM, Ramchandra Prasad MV, Kathariya R, Patel SP, Sharma A (2011) Gingival crevicular fluid and serum visfatin concentration: their relationship in periodontal health and disease. J Periodontol 82, 1314-1319.

19. Culshaw S, McInnes IB, Liew FY (2011) What can the periodontal community learn from the pathophysiology of rheumatoid arthritis? J Clin Periodontol 38, Suppl 11, 106-113.

20. Silness J, Löe H (1964) Periodontal disease in pregnancy. II.
Correlation between oral hygiene and periodontal condition. Acta Odontol Scand 22, 121-135.

21. Ainamo J, Bay I (1975) Problems and proposals for recording gingivitis and plaque. Int Dent J 25, 229-235.

22. Löe H, Silness J (1963) Periodontal disease in pregnancy. I. Pevalence and severity. Acta Odontol Scand 21, 533-551.

23. Schwarz F, Bieling K, Latz T, Nuesry E, Becker J (2006) Healing of intrabony peri-implantitis defects following application of a nanocrystalline hydroxyapatite (Ostim) or a bovine-derived xenograft (Bio-Oss) in combination with a collagen membrane (Bio-Gide). A case series. J Clin Periodontol 33, 491-499.

24. Griffiths GS (2003) Formation, collection and significance of gingival crevice fluid. Periodontol 2000 31, 32-42.

25. Luk T, Malam Z, Marshall JC (2008) Pre-B cell colonyenhancing factor $(\mathrm{PBEF})$ /visfatin: a novel mediator of innate immunity. J Leukoc Biol 83, 804-816.

26. Seymour GJ, Ford PJ, Cullinan MP, Leishman S, Yamazaki K (2007) Relationship between periodontal infections and systemic disease. Clin Microbiol Infect 13, Suppl 4, 3-10.

27. McCulloch CA (1994) Host enzymes in gingival crevicular fluid as diagnostic indicators of periodontitis. J Clin Periodontol 21, 497-506.

28. Kinney JS, Morelli T, Oh M, Braun TM, Ramseier CA, Sugai JV et al. (2014) Crevicular fluid biomarkers and periodontal disease progression. J Clin Periodontol 41, 113-120.

29. Buduneli N, Kinane DF (2011) Host-derived diagnostic markers related to soft tissue destruction and bone degradation in periodontitis. J Clin Periodontol 38, Suppl 11, 85-105.

30. Lamster IB, Oshrain RL, Fiorello LA, Celenti RS, Gordon JM (1988) A comparison of 4 methods of data presentation for lysosomal enzyme activity in gingival crevicular fluid. J Clin Periodontol 15, 347-352.

31. Lin SJ, Chen YL, Kuo MY, Li CL, Lu HK (2005) Measurement of gp130 cytokines oncostatin M and IL-6 in gingival crevicular fluid of patients with chronic periodontitis. Cytokine 30, 160-167.

32. Tsai CC, Ho YP, Chen CC (1995) Levels of interleukin-1 beta and interleukin-8 in gingival crevicular fluids in adult periodontitis. J Periodontol 66, 852-859.

33. Buduneli N, Özçaka Ö, Nalbantsoy A (2011) Salivary and plasma levels of toll-like receptor 2 and Toll-like receptor 4 in chronic periodontitis. J Periodontol 82, 878-884.

34. Mohamed HG, Idris SB, Mustafa M, Ahmed MF, Åstrøm AN, Mustafa K et al. (2015) Impact of chronic periodontitis on levels of glucoregulatory biomarkers in gingival crevicular fluid of adults with and without type 2 diabetes. PLoS One 10, e0127660.

35. Tabari ZA, Azadmehr A, Nohekhan A, Naddafpour N, Ghaedi FB (2014) Salivary visfatin concentrations in patients with chronic periodontitis. J Periodontol 85, 1081-1085.

36. Özcan E, Saygun NI, Serdar MA, Kurt N (2015) Evaluation of the salivary levels of visfatin, chemerin, and progranulin in periodontal inflammation. Clin Oral Investig 19, 921-928.

37. Raghavendra NM, Pradeep AR, Kathariya R, Sharma A, Rao 
NS, Naik SB (2012) Effect of non surgical periodontal therapy on gingival crevicular fluid and serum visfatin concentration in periodontal health and disease. Dis Markers 32, 383-388.

38. Wu Y, Chen L, Wei B, Luo K, Yan F (2015) Effect of nonsurgical periodontal treatment on visfatin concentrations in serum and gingival crevicular fluid of patients with chronic periodontitis and type 2 diabetes mellitus. J Periodontol 86, 795-800.

39. Abolfazli N, Jabali S, Saleh Saber F, Babaloo Z,
Shirmohammadi A (2015) Effect of non-surgical periodontal therapy on serum and salivary concentrations of visfatin in patients with chronic periodontitis. J Dent Res Dent Clin Dent Prospects 9, 11-17.

40. Özcan E, Saygun NI, Serdar MA, Kubar A, Bengi VU (2016) Porphyromonas gingivalis and epstein-barr virus are associated with increased levels of visfatin in gingival crevicular fluid. J Periodontol 87, 443-451. 